\title{
Importância de tecnologia educacional para usuários submetidos a cineangiocoronariografia
}

\author{
Importance of educational technology for users submitted to cineangiocoronariography \\ Importancia de tecnología educativa para usuários sometidos a cineangiocoronariografía
}

Recebido: 19/10/2021 | Revisado: 30/10/2021 | Aceito: 06/11/2021 | Publicado: 10/11/2021

\author{
Rosana Moreira Sant'Anna \\ ORCID: https://orcid.org/0000-0001-7817-1895 \\ Universidade Federal Fluminense, Brasil \\ E-mail: vifelipe@id.uff.br \\ Vitória Meireles Felipe de Souza \\ ORCID: https://orcid.org/0000-0002-1129-6324 \\ Universidade Federal Fluminense, Brasil \\ E-mail: vifelipe@id.uff.br \\ Rafael Pires da Silva \\ ORCID: https://orcid.org/0000-0002-9786-3229 \\ Universidade Federal Fluminense, Brasil \\ E-mail: Rafael.pires.silva72@gmail.com \\ Harlon França de Menezes \\ ORCID: https://orcid.org/0000-0001-9884-6511 \\ Universidade Federal Fluminense, Brasil \\ E-mail: harlonmenezes@ hotmail.com \\ Alessandra Conceição Leite Funchal Camacho \\ ORCID: https://orcid.org/0000-0001-6600-6630 \\ Universidade Federal Fluminense, Brasil \\ E-mail: cicacamacho@gmail.com \\ Marina Sant'Anna Silva \\ ORCID: https://orcid.org/0000-0002-5467-1235 \\ Universidade Federal do Rio de Janeiro, Brasil \\ E-mail: mss.santsilva@gmail.com
}

\begin{abstract}
Resumo
Objetivo: refletir sobre a importância de estratégia educativa no processo de construção de tecnologia educacional para orientação na assistência de usuários submetidos ao exame de cineangiocoronariografia. Conteúdo: entre as diversas competências da Enfermagem, a educação em saúde se mostra como prática efetiva nas diversas atribuições a ela destinada, de forma holística reconhece o assistir dentro da concepção de integralidade onde se propõe um cuidado centrado na dimensão física, mental, emocional e espiritual. As fontes principais para este estudo foram as relações estabelecidas entre doenças crônicas não transmissíveis, tecnologia educativa e processo dialógicoreflexivo em educação em saúde. Conclusão: o processo de educação em saúde requer que seja levado em conta as interpretações ou apreensões do contexto e estas envolvem as crenças, valores, experiências, classe social, religião e cultura de cada usuário. A intervenção educativa na assistência promove melhor qualidade à clientela a ser assistida, oferecendo possibilidade esclarecedora sobre o exame.
\end{abstract}

Palavras-chave: Tecnologia educacional; Cateterismo cardíaco; Cuidados de enfermagem; Qualidade de vida.

\begin{abstract}
Objective: to reflect on the importance of an educational strategy in the process of building educational technology for guidance in assisting users undergoing coronary angiography. Content: among the various competences of Nursing, health education is shown as an effective practice in the various attributions assigned to it, in a holistic way it recognizes care within the concept of comprehensiveness, which proposes a care centered on the physical, mental, emotional, and spiritual. The main sources for this study were the relationships established between noncommunicable chronic diseases, educational technology, and the dialogical-reflective process in health education. Conclusion: the health education process requires considering the interpretations or apprehensions of the context and these involve the beliefs, values, experiences, social class, religion and culture of each user. The educational intervention in assistance promotes better quality to the clientele to be assisted, offering clarifying possibilities about the exam.
\end{abstract}

Keywords: Educational technology; Cardiac catheterization; Nursing care; Quality of life. 


\begin{abstract}
Resumen
Objetivo: reflexionar sobre la importancia de una estrategia educativa en el proceso de construcción de tecnología educativa para orientar en la asistencia a los usuarios sometidos a coronariografía. Contenido: entre las diversas competencias de la Enfermería, la educación para la salud se muestra como una práctica efectiva en las distintas atribuciones que se le asignan, de manera holística reconoce el cuidado dentro del concepto de integralidad, que propone un cuidado centrado en lo físico, mental, emocional. y espiritual. Las principales fuentes de este estudio fueron las relaciones que se establecen entre las enfermedades crónicas no transmisibles, la tecnología educativa y el proceso dialógico-reflexivo en la educación para la salud. Conclusión: el proceso de educación en salud requiere tomar en cuenta las interpretaciones o aprehensiones del contexto y estas involucran las creencias, valores, vivencias, clase social, religión y cultura de cada usuario. La intervención educativa en asistencia promueve una mejor calidad a la clientela a ser asistida, ofreciendo posibilidades esclarecedoras sobre el examen.
\end{abstract}

Palabras clave: Tecnología educacional; Cateterismo cardíaco, Atención de enfermeira; Calidad de vida.

\title{
1. Introdução
}

As doenças crônicas não transmissíveis (DCNT) constituem-se em problema de saúde pública com prevalência nos grandes centros contribuindo significativamente para o aumento das taxas de mortalidade em todas as regiões brasileiras. Segundo a Organização Pan Americana da Saúde (Ferreira et al, 2017, Freire et al, 2017) ao longo da última décadas se tornaram as principais causas de mortalidade em todo o mundo, representando cerca de $70 \%$ de todas as mortes. Elas constituem-se como uma das principais causas de permanência hospitalar prolongada sendo responsáveis por grande parte dos casos de incapacidade e morbimortalidade promovendo forte impacto clínico e financeiro no Brasil e no mundo (Levine et al, 2016).

É de suma importância ressaltar o controle das DCNT, que deve ser evidenciado de forma global. A Organização das Nações Unidas, em assembleia geral em 2011, reuniu chefes de estados e países com a finalidade de assumirem compromissos globais para o seu enfrentamento. Entre as metas e acordos a serem buscados intensificou-se a redução na mortalidade prematura por doenças não transmissíveis e redução dos fatores de risco, como o tabagismo, a inatividade física e alcoolismo (Malta, Oliveira, Santos, Andrade, \& Silva, 2016).

O Ministério da Saúde no Brasil através do plano de ações estratégicas para o enfrentamento das DCNT, visou preparar o país para que elas sejam enfrentadas e detidas no período preliminar de dez anos, entre 2011 e 2022 , através de ações no sentido da estruturação e operacionalização de um sistema específico de vigilância para as doenças e agravos não transmissíveis (Ministério da Saúde, 2016).

Dentre os objetivos desse plano de enfrentamento, o Ministério da Saúde buscou proporcionar à população alternativas relativas à construção de comportamentos saudáveis ao longo da vida. As DCNT têm fatores de risco que são comuns às quatro doenças crônicas de maior impacto mundial, causadoras de $72 \%$ das causas de mortes: o tabaco, a alimentação não saudáveis, a inatividade física, o consumo excessivo de álcool, que são passíveis de prevenção (Ministério da Saúde, 2016, Ferreira et al, 2017).

O acompanhamento das tendências das DCNT bem como seus fatores de risco torna-se necessário para o monitoramento, objetivando-se identificar as vulnerabilidades sociais e possibilidades dentro do campo da saúde para o seu enfrentamento (Malta, Oliveira, Santos, Andrade, \& Silva, 2016). A mudança dessa realidade está aliada às mudanças no estilo de vida com vistas a diminuição do impacto da doença e a melhoria na qualidade de vida do usuário (Ministério da Saúde, 2016).

Entre as DCNT, as doenças cardiovasculares ocupam um patamar alto em relação a morbimortalidade da população. As intervenções para a sua prevenção e seu controle incluem diversas ações, dentre elas a educativa. A construção de estratégias preventivas para o seu enfrentamento possibilita valorização da dimensão subjetiva e social em todas as práticas de atenção e gestão em saúde, dando suporte aos usuários que diariamente frequentam o serviço em busca de marcação e 
realização dos procedimentos de cineangiocoronariografia, criando assim uma atuação profissional capaz de acolher, escutar e responder às inquietações e dúvidas dessa clientela.

É papel do enfermeiro a orientação, ensino, avaliação e acompanhamento embasado em dados confiáveis, possibilitando o cuidado adequado do usuário (Matias, 2016). A enfermagem, inserida na equipe multiprofissional de saúde, deverá estar atenta para a avaliação e o cuidado dos pacientes submetidos ao exame de cineangiocoronariografia. $\mathrm{O}$ aprimoramento de conhecimentos e técnicas em enfermagem embasados em cardiologia intervencionista contribui para que o enfermeiro tenha um olhar mais atento para as necessidades e dificuldade desta clientela. O cuidado humanizado deverá estar associado ao nível de complexidade apresentado, como também as diferentes tecnologias a serem utilizadas nessa assistência (Costa, Cardoso, \& Silva, 2019).

A cineangiocoronariografia é um exame cardiológico invasivo que se constitui na introdução de finos cateteres nas artérias, através da punção ou dissecção de uma veia ou artéria periférica, seguida da administração de contraste radiológico. Com esse exame, se possibilita o diagnóstico através da medição da pressão e gradientes de pressão, mas também se faz o tratamento das estenoses das válvulas cardíacas, isquemias coronárias, por meio da desobstrução mecânica dos vasos ou da introdução de stents (Barretta, Auda, Barancelli, \& Antoniolli, 2017).

O uso de tecnologias como intervenção nas ações em educação em saúde favorece o processo educativo do usuário, a tecnologia educacional deve possibilitar dimensões interacionais que permitam aos profissionais a utilização dos sentidos para a escolha e a realização da assistência a fim de (re)encontrar a sensitividade, a solidariedade, o amor, a ética e o respeito de si e do outro (clientela), promovendo o apoio, a manutenção e a promoção do processo da vida (Freire, 2017).

Portanto o objetivo deste estudo foi refletir sobre a importância da utilização de estratégias educativas no processo de construção de tecnologia educacional de orientação a assistência de usuários portadores de doenças cardiovasculares submetidos ao exame de cineangiocoronariografia.

\section{Metodologia}

Trata-se de um estudo descritivo, exploratório com abordagem qualitativa, que procura explicar os meandros das relações sociais - consideradas essência e resultado da atividade humana criadora, afetiva e racional, e que podem ser apreendidas através do cotidiano, da vivência e da explicação do senso comum (Urquiza \& Marques, 2016).

Diante disso, o presente estudo trata-se de uma reflexão teórica sendo desenvolvida a partir de teorias, abordagens e conceitos disseminados e consolidados por outros pesquisadores que abordam a temática a fim de sustentar esta reflexão (Barros, Formiga \& Vasconcelos, 2021), associados a pesquisa realizada no Serviço de Hemodinâmica do Hospital Universitário, vinculado à Universidade Federal Fluminense, no estado do Rio de Janeiro.

O laboratório de Hemodinâmica é um espaço dos serviços de saúde onde ocorrem procedimentos invasivos que possibilitam o estudo hemodinâmico cardíaco e arterial dos usuários. Esses exames têm por objetivo realizar diagnóstico e tratamento adequado de cardiopatias e arteriopatias.

Os participantes do estudo foram 20 usuários que procuraram o serviço de hemodinâmica para marcação e realização do exame de cineangiocoronariografia e que aceitaram participar do estudo, realizado no período de outubro a dezembro de 2013. Os critérios de inclusão dos participantes foram: usuários de ambos os sexos, idade maior ou igual a 18 anos, atendidos ambulatorialmente e ou internados, e que estivessem sob cuidados médicos. Os critérios de exclusão foram: usuários submetidos ao exame em caráter de urgência ou mentalmente comprometidos.

A reflexão parte da produção dos dados constituídos por meio de entrevista semiestruturada durante a admissão do usuário nas unidades de atendimento ou de internação. Utilizou-se de três instrumentos para a coleta de dados, um contemplando o perfil sociodemográficos dos usuários atendidos no serviço de HD, o segundo que possibilitou a identificação 
dos conhecimentos e crenças relacionados ao exame realizado e o terceiro onde os usuários avaliaram a cartilha educativa construída a partir das evidências cientificas que emergiram do estudo.

As entrevistas foram gravadas e transcritas na íntegra, para posterior análise (Urquiza \& Marques, 2016). A saturação dos dados foi o critério para encerramento da amostra. Os dados foram analisados e interpretados a partir da triangulação dos achados, à luz da análise temática de conteúdo (Urquiza \& Marques, 2016). A pesquisa atende a Res. 466/2012, n. 429.165 .

\section{Resultados e Discussão}

O cuidar em enfermagem é uma atividade singular porque visualiza o usuário como um todo. Engloba o indivíduo com sua cultura, seus princípios e valores, sua religião ou filosofia de vida, condensando a situação socioeconômica ao seu estado emocional e biológico, ou seja, o cuidar holístico (Veras, Menezes, Guerrero-Castañeda, Soares, \& Anton Neto, 2019).

A enfermagem dentro do contexto da saúde é resultado do momento histórico de cada povo que carrega em si suas crenças, religião e costumes. Não compreende apenas o cuidar e curar doentes - é na realidade um processo que caminha junto ao patamar de acessibilidade, na qual a conscientização de que o outro carrega em si uma trajetória de vida, que não pode ser deixada de lado (Miranda, Arruda, \& Melo, 2019). A educação em saúde envolve o processo educativo que coloca o cotidiano no trabalho. Consiste na atualização cotidiana das práticas segundo os aportes teóricos, metodológicos, científicos e tecnológicos disponíveis para a construção de relações e processos (Ferreira, 2019).

Este processo caminha lado a lado com o acolher, abrindo-se espaços possíveis de escuta, onde o outro pode se expor, se abrir, se deixar conhecer e ser tratado, sem reservas ou medos. Visando esta integralidade, a enfermagem quer utilizar-se de ações que possibilitam o atendimento e a assistência das necessidades desses usuários perante o processo saúde/doença, cujo olhar centra-se na totalidade do indivíduo, ampliando e respeitando-se as dimensões a serem assistidas, ou seja, as dimensões biológicas, psicológicas e sociais presentes em cada indivíduo (Chaves et al, 2017, Ministério da Saúde, 2004).

As orientações desenvolvidas pelo enfermeiro, precisam estar voltadas para o processo de educação em saúde. Ele tem na sua formação profissional condições de instrumentalizar ações educativas para a saúde, visando a adesão ao procedimento. É exigido desse profissional compromisso, competência e preocupação no que se refere à possibilidade de colaborar para que usuários/família tenham minimizado o estresse que é causado pela cineangiocoronariografia, bem como diminuir o medo e a ansiedade ocasionados pela realização e resultado do procedimento (Anna, Escudeiro, Ferreira, Teixeira, \& Castelo Branco, 2016).

A participação dos usuários no processo da construção da cartilha é recomendada como uma abordagem inclusiva e coletiva. A construção de materiais educativos deve levar em consideração as representações sociais dos atores envolvidos no processo de se educar enquanto fenômeno psicossocial (Freire, 2017). Saber que no espaço público estão radicadas representações que contradizem, confrontam, veiculam e reiteram o desenvolvimento de práticas interativas direcionadas a aquisição, promoção e prevenção da saúde. Assistir de forma individualizada sem perder o foco do social, constitui o processo pelo qual os profissionais precisam ser instrumentalizados e motivados para tal (Jodolet, 2018).

Recomenda-se ainda que a interação entre as pessoas envolvidas nesse processo leve em consideração os processos emocionais, culturais, afetivos e intelectuais de forma que possam intervir ou mudar ou ainda proporcionar mudanças de condutas e ou comunicações sociais. Os indivíduos não são apenas processadores de informações, nem meros portadores de ideologias ou crenças coletivas (Freire, 2017). Dessa forma, são pensadores ativos que produzem e comunicam suas próprias representações e soluções para as questões que colocam a si mesmos (Azevendo, \& Modesto, 2016).

Estudos demonstram que usuários agendados para qualquer exame diagnóstico invasivo, considerando-se, ainda, a condição de cronicidade das doenças que apresentem, mostram as mesmas inquietações: ansiedade relacionada à ameaça da 
integridade física e ao bem-estar, como também às implicações advindas de um resultado diagnóstico inconclusivo ou positivo (Costa, Cardoso, \& Silva, 2019), nesta ótica, ao fornecer orientações acerca do exame a se realizado constitui-se em possibilidade de minimizar essas inquietações.

$\mathrm{O}$ medo e a ansiedade costumam estar relacionados aos efeitos imediatos da doença e do tratamento, as reações de afastamento impostas pelas mudanças de papéis, a problemas sociais e psicológicos preexistentes (Anna, Escudeiro, Ferreira, Teixeira, \& Castelo Branco, 2016). As patologias que atingem o coração podem trazer a necessidade radical de mudança de vida, acrescente-se a isso o fato de que a morte, muitas vezes, não é elaborada de forma consciente o medo, muitas vezes pode não ser expresso por palavras (Jodelet, 2018). O adoecimento, portanto, é um fenômeno multidimensional e complexo, que envolve não apenas eventos físicos, mas também fatores psicológicos, resultando em processos de aprendizagem social e cultural em meio as relações vivenciadas (Pompeo, Eid, Carvalho, Bertolli, \& Oliveira, 2017).

É importante o envolvimento do usuário nesse processo, "visto que o conhecimento não é algo definitivo, nem acabado, todo dia existe algo para se aprender e apreender, pois [...] aprender é construir, reconstruir, constatar para mudar [...]" (Freire, 2017, Messias, 2016, Barretta, Auda, Barancelli, \& Antoniolli, 2017). As ações a serem administradas nas orientações precisam ser embasadas não só no processo assistencial pois o conhecimento que se estima para o usuário precisa atender as suas necessidades de orientações referentes à cineangiocoronariografia e nas áreas de promoção de saúde salientadas por ele (Teixeira, 2011).

No contexto da Enfermagem as tecnologias estão sendo produzidas, validadas e avaliadas. Verificam-se estudos e estratégias voltadas para tecnologias educacionais reforçando que são fomentos úteis a democratização do saber, contribuindo para aproximar a prática e a teoria (Teixeira, 2011). A tecnologia educativa pode ser considerada como produto das tecnologias assistenciais, as quais incluem a construção de um saber técnico-científico resultante de investigações, aplicações de teorias e da experiência cotidiana dos profissionais e da clientela, constituindo-se, portanto, em um conjunto de ações sistematizadas, processuais e instrumentais para a prestação de assistência qualificada ao ser humano em todas as suas dimensões: o ser físico, o ser psíquico, o ser espiritual, o ser social, o ser intelectual, o ser que pensa, sente, aspira e deseja, o ser que age, o ser de relações, o ser particular e coletivo (Vieira, Alvarez, Sena, \& Fagundes, 2016, Berardinelli, Guedes, Ramos, \& Silva, 2015).

Os materiais educativos impressos na área de saúde constituem uma prática comum utilizada pelo SUS (Ministério da Saúde, 2014, Costa et al, 2018) usualmente são empregados manuais de cuidados em saúde, folhetos e cartilhas e estes precisam estar associados às formas de comunicação e diálogo que promovam uma relação dialógica (Freire, 2017). A criação e o desenvolvimento de um material educativo precisam ser diversificados a fim de promover o conhecimento através de uma leitura que não seja exaustiva e maçante, mas ao contrário, que seja capaz de envolver o usuário nesse processo de corresponsabilidade pela sua saúde (Leite et al, 2018).

A realidade do usuário portador de doença cardiovascular submetido ao exame de cineangiocoronariografia bem como a necessidade da realização desse exame vai determinar o papel estratégico da tecnologia educativa a ser utilizada como suporte a aqueles que precisam ter suas questões de saúde/doença resolvidas. O enfermeiro em sua práxis pode produzir e revolucionar na questão do seu papel social enquanto fomento para a educação em saúde (Freire, 2017).

\section{Conclusão}

O enfermeiro tem papel de grande importância no cuidado em saúde, pois é um profissional altamente qualificado, com preparação técnica, que está apto para desenvolver atividades educativas em saúde. É necessário a tomada de consciência, de que precisa fazer uso da prática embasada em evidências científicas para assim propiciar uma assistência que não seja fragmentada. 
As orientações em saúde precisam estar direcionadas para as necessidades dos usuários, além dos conceitos técnicoscientíficos, dar ênfase às relações. As contribuições da construção de tecnologia educativa para usuários submetidos a cineangiocoronariografia se voltam para a redução do nível de estresse, medo e ansiedade perante o exame e melhoria na qualidade de vida a partir do compartilhamento de saberes, esclarecimento acerca das ações de promoção, prevenção e recuperação da saúde.

Através da educação em saúde do usuário, no momento da marcação ou no período que antecede o exame, o enfermeiro tem a possibilidade de reduzir o nível de ansiedade utilizando-se além das orientações, tecnologia educativa que vai adicionar à assistência condições ideais para o cuidar, otimizando assim o fluxo de informações.

Encontrar a melhor maneira de possibilitar o cuidado, a adesão, de forma que as experiências somadas (profissionalusuário) contribuam no contexto vivido por ambos para a integralidade no processo de educar-cuidar. A assistência de saúde torna-se qualificada, quando se é realizada com base nas necessidades dos usuários, na criação de espaços que favoreçam o processo terapêutico do cuidar, onde se priorize o diálogo e a escuta, onde suas inquietações são tomadas a sério.

Em educação em saúde é necessário frisar que todos os esforços precisam estar centrados em se prevenir, promover e se recuperar a saúde. Dentro das competências do enfermeiro a educação em saúde torna-se um campo de suma importância ao desenvolvimento social, contribuindo junto com as práticas educativas para a ampliação dos saberes. Permite também a abordagem interdisciplinar, integralizando a assistência pela proximidade do usuário-profissional, de maneira a criar e garantir a humanização da assistência, desta forma o compartilhamento de saberes, o diálogo e a escuta são contemplados, e se coloca em prática o que o Sistema Único de Saúde preconiza.

Os benefícios advindos dessa prática educativa estarão associados a: aquisição e produção de novos conhecimentos, reflexão sobre qualidade de vida e mudanças de hábitos não saudáveis. A temática tecnologia educativa tem se mostrado pertinente e atual, sendo assim, sugere-se que estudos dialógicos, acadêmicos e profissionais sejam contemplados, para que as orientações ocorram de forma a prestigiar as necessidades dos usuários em saúde.

Novos estudos precisam ser realizados e colocados em prática visando a utilização de estratégias que sejam adequadas aos usuários submetidos a cineangiocoronariografia. O uso dessas tecnologias deve proporcionar esclarecimentos, ampliar e possibilitar a aprendizagem e promover a aquisição de hábitos saudáveis que impactem na qualidade de vida. Esse comprometimento deve ser direcionado a todo profissional de saúde que atenda a essa clientela.

\section{Referências}

Anna, R. M. S., Escudeiro, C. L., Ferreira, S. C. M., Teixeira, M. L. O., \& Castelo Branco, E. M. S. (2016). Tecnologia Educativa Em Saúde Para Usuários Da Hemodinâmica. Journal of Nursing UFPE/Revista de Enfermagem UFPE, 10(10).

Azevedo, P. A. C., \& Modesto, C. M. S. (2016). A (ré)organização do núcleo de cuidado familiar diante das repercussões da condição crônica por doença cardiovascular. Saúde em Debate, 40(110): 183-194.

Barretta, J. C., Auda, J., Barancelli, M. D. C., \& Antoniolli D. (2017). Post-operative in cardiac surgery: reflecting about nursing care. Rev Fund Care Online, 9(1): 259-264.

Barros, MB, Formiga, N. S, \& Vasconcelos, C. R. M. (2021). Uma reflexão teórica sobre a confiança nas relações interpessoais em ambientes organizacionais. Research, Society and Development, 10(5): e53610515299

Berardinelli, L., Guedes, N., Ramos, J., \& Silva, M. (2015). Educational technology as a strategy for the empowerment of people with chronic illnesses. Revista enferm UERJ, 22(5), 603-609.

Brasil. Ministério da Saúde. (2004). Política Nacional de Humanização. O acolhimento como estratégia de interferência nos processos de trabalho.

Brasil. Ministério da Saúde. (2014). Educação Permanente em Saúde: Reconhecer a produção local de cotidianos de saúde e ativar práticas colaborativas de aprendizagem e de entrelaçamento de saberes. Secretaria de Gestão Estratégica do Trabalho e da Educação em Saúde.

Brasil. Ministério da Saúde. Secretária de vigilância em Saúde. Departamento de Análise de Situação de Saúde. Plano de ações estratégicas para o enfrentamento das doenças crônicas não transmissíveis (DCNT) no brasil 2011-2022.

Chaves et al. (2017). Nursing supervision for care comprehensiveness. Revista Brasileira de Enfermagem, 70(5): $1106-1111$. 
Costa, et al. (2018). Construction and validation of educational materials for children with chronic disease: an integrative review. Revista enferm UERJ, 26: e34208.

Costa, M. S., Cardoso, L. G. S., \& Silva, S. M. (2019). Conhecimento dos enfermeiros sobre ações de enfermagem e complicações em procedimentos invasivos coronarianos. Arq Med Hosp Fac Cienc Med Santa Casa São Paulo, 64(2):76-83.

Ferreira et tal. (2017). Fatores de risco para doenças cardiovasculares em idosos. Rev enferm UFPE, 11(12):4895-905.

Ferreira, R. G. S. (2019). Percepções dos enfermeiros acerca das ações de educação continuada nos cenários da prática profissional. Enfermagem Brasil, 17(5): 504-510.

Freire, et al. (2017). Panorama no Brasil das doenças cardiovasculares dos últimos quatorze anos na perspectiva da promoção à saúde. Rev Saúde Desenvol, 1(9):21-44.

Freire, P. (2017). Pedagogia do Oprimido. Editora Paz \& Terra.

Jodelet, D. (2018). Ciências sociais e representações: estudo dos fenômenos representativos e processos sociais, do local ao global. Sociedade e Estado, 33(2): 423-442.

Leite et al. (2018). Construction and validation of an Educational Content Validation Instrument in Health. Revista Brasileira de Enfermagem, 71 (4): 16351641 .

Levine, et al. 2016. ACC/AHA guideline focused update on duration of dual antiplatelet therapy in patients with coronary artery disease: A report of the American College of Cardiology/American Heart Association Task Force on Clinical Practice Guidelines: An update of the 2011 ACCF/AHA/SCAI guideline for percutaneous coronary intervention, 2011 ACCF/AHA guideline for coronary artery bypass graft surgery, 2012 ACC/AHA/ACP/AATS/PCNA/SCAI/STS guideline for the diagnosis and management of patients with stable ischemic heart disease, 2013 ACCF/AHA guideline for the management of ST-elevation myocardial infarction, 2014 AHA/ACC guideline for the management of patients with non-ST-elevation acute coronary syndromes, and 2014 ACC/AHA guideline on perioperative cardiovascular evaluation and management of patients undergoing noncardiac surgery. Circulation., 134:e123-55.

Malta, D. C., Oliveira, T. P., Santos, M. A. S., Andrade, S. S. C. A., \& Silva, M. M. A. (2017). Progress with the Strategic Action Plan for Tackling Chronic Non-Communicable Diseases in Brazil, 2011-2015. Epidemiol. Serv. Saúde, 25(2): 373-390.

Matias, C. P. P. (2016). Pedagogia da autonomia: saberes necessários à prática educativa. Criar Educ, 5(2):1-5.

Miranda, A. V. S., Arruda, M. P., \& Melo, S. M. M. (2019). Paradigma do cuidado complexo em saude: produção de conhecimento no campo da enfermagem. EDU, 8(3):27-40.

Pompeo, D. A., Eid, L. P., Carvalho IG, Bertolli ES, \& Oliveira NS. (2017). Autoestima de pacientes com doença arterial coronariana. Rev Rene, 18(6):71219.

Teixeira, E., \& Mota, V. M. S. S. (2011). Tecnologias educacionais em foco. São Caetano do Sul, SP: Difusão Editora. Serie Educação em Saúde.

Urquiza, M. A., \& Marques, D. B. (2016). Análise de conteúdo em termos de Bardin aplicada à comunicação corporativa sob o signo de uma abordagem teórico empírica. Entretextos, Londrina, 16(1):115-144.

Veras, S. M. C. B., Menezes, T. M. O., Guerrero-Castañeda, R. F., Soares, M. V., \& Anton Neto, F. R. (2019). Nurse care for the hospitalized elderly's spiritual dimension. Revista Brasileira de Enfermagem, 72(2): 236-242.

Vieira, G. B., Alvarez, A. M., Sena, A. C., \& Fagundes, M. A. F. (2016). O conhecimento da pessoa idosa sobre seus direitos de acesso ao cuidado em saúde. Cienc. Cuid. Saúde, 14(4):1528 -1536. 